\title{
Philosophiques
}

\section{De Popkin à Rousseau : retour sur le scepticisme des Lumières}

\section{Sébastien Charles}

Volume 35, numéro 1, printemps 2008

Les valeurs de l'ironie (1) et le scepticisme à l'âge classique (2)

URI : https://id.erudit.org/iderudit/018250ar

DOI : https://doi.org/10.7202/018250ar

Aller au sommaire du numéro

\section{Éditeur(s)}

Société de philosophie du Québec

\section{ISSN}

0316-2923 (imprimé)

1492-1391 (numérique)

Découvrir la revue

\section{Citer cet article}

Charles, S. (2008). De Popkin à Rousseau : retour sur le scepticisme des Lumières. Philosophiques, 35(1), 275-290. https://doi.org/10.7202/018250ar

\section{Résumé de l'article}

Le scepticisme des Lumières est le parent pauvre des études que Popkin a consacrées au scepticisme moderne. En général, Popkin ne confère au scepticisme des Lumières qu'une valeur propédeutique ou méthodologique. Si Popkin est revenu à plusieurs reprises sur ce portrait qu'il a brossé du scepticisme des Lumières pour le nuancer, évoquant notamment les travaux de Baker, d'Olaso ou de Tonelli, c'est pour accepter que le scepticisme ait été plus prégnant au XVIII ${ }^{\mathrm{e}}$ siècle qu'il ne le pensait, mais sans pour autant modifier son sentiment quant à l'usage qui en fut fait. Or, à partir de l'évocation de formes de scepticisme délaissées par Popkin (le scepticisme clandestin, l'égoïsme), cet article propose une réévaluation de l'ensemble du scepticisme des Lumières et en teste la validité en prenant Rousseau pour exemple. L'avantage du recours à Rousseau, c'est que ce dernier a connu à la fois le scepticisme clandestin et l'égoïsme, et qu'il est donc à même de montrer l'influence de ce scepticisme souterrain sur les Lumières dans leur ensemble. 


\title{
De Popkin à Rousseau : retour sur le scepticisme des Lumières
}

\author{
SÉBASTIEN CHARLES \\ Université de Sherbrooke \\ Sebastien.Charles@USherbrooke.ca
}

\begin{abstract}
RÉSUMÉ. - Le scepticisme des Lumières est le parent pauvre des études que Popkin a consacrées au scepticisme moderne. En général, Popkin ne confère au scepticisme des Lumières qu'une valeur propédeutique ou méthodologique. Si Popkin est revenu à plusieurs reprises sur ce portrait qu'il a brossé du scepticisme des Lumières pour le nuancer, évoquant notamment les travaux de Baker, d'Olaso ou de Tonelli, c'est pour accepter que le scepticisme ait été plus prégnant au XVIII ${ }^{e}$ siècle qu'il ne le pensait, mais sans pour autant modifier son sentiment quant à l'usage qui en fut fait. Or, à partir de l'évocation de formes de scepticisme délaissées par Popkin (le scepticisme clandestin, l'égoïsme), cet article propose une réévaluation de l'ensemble du scepticisme des Lumières et en teste la validité en prenant Rousseau pour exemple. L'avantage du recours à Rousseau, c'est que ce dernier a connu à la fois le scepticisme clandestin et l'égoïsme, et qu'il est donc à même de montrer l'influence de ce scepticisme souterrain sur les Lumières dans leur ensemble.
\end{abstract}

ABSTRACT. - The scepticism of the Enlightenment was neglected in the studies that Popkin devoted to early modern scepticism. Generally, Popkin only attributes a preparatory, or methodological role to the scepticism of the Enlightenment. Indeed, Popkin himself has come back several times to the picture he had drawn of the Enlightenment, notably in light of the works by Baker, Olaso or Tonelli. However, it was only to accept that scepticism was more developed than he had first thought, and never to change his mind about the influence that it had on this time-period. Through the analysis of forms of scepticism that were totally neglected by Popkin (i.e., clandestine scepticism and egoism), this article proposes a complete re-evaluation of scepticism in the Enlightenment. Rousseau is particularly interesting here because he has known both the clandestine scepticism, and the egoism movement. This article takes him as a case study in order to test the validity of its thesis concerning the influence of this underground scepticism on the Enlightenment as a whole.

Dans un article intitulé « Scepticism in the Enlightenment » paru en 1963 dans les Studies on Voltaire and the Eighteenth Century, Richard Popkin avance l'idée selon laquelle «il y avait, et ce de manière quelque peu surprenante, très peu de scepticisme au siècle des Lumières ${ }^{1} »$ — du moins si l'on considère le scepticisme sous sa forme philosophique. Trois grands courants philosophiques ont été les représentants de ce pyrrhonisme fort discret, en rien comparable à la vigueur sceptique du siècle précédent : le premier s'inscrit dans la filiation montanienne et baylienne, le deuxième représente une forme de fidéisme

1. Popkin (1997), p. 1 (traduction nôtre).

PHILOSOPHIQUES 35/1 — Printemps 2008, p. 275-290 
irrationaliste qui anticipe par certains côtés les thèses développées par la suite par Kierkegaard, et le troisième renvoie à un type de scepticisme épistémologique caractéristique des premiers adversaires du criticisme kantien au sein de l'Académie de Berlin. Mais, pour Popkin, ces courants n'ont pas connu de développement d'envergure et il faudrait plutôt parler à leur égard de scepticisme souterrain, dont l'activité sur la scène intellectuelle des Lumières fut faible, sinon nulle. Le scepticisme des Lumières se réduirait en réalité à la figure emblématique de Hume, mais cette réduction n'aurait été valide que si le scepticisme de ce dernier avait été considéré à sa juste valeur. Or, on sait que pour les philosophes des Lumières, la réputation de Hume fut plus fondée sur ses travaux d'historien que de philosophe. Dès lors, Popkin s'est cru autorisé à écrire que "Hume, à titre de sceptique, fut un homme complètement isolé dans le gai tourbillon social des salons parisiens ${ }^{2} »$.

Bien évidemment, Popkin ne nie pas l'existence d'une influence réelle du scepticisme dans le premier quart du XVIII ${ }^{\text {e }}$ siècle français, qui se manifeste à la fois à travers les écrits de Pierre Bayle (et notamment son fameux Dictionnaire, de nombreuses fois réédités dans les premières années du siècle), la publication posthume en 1721 du Traité de la foiblesse de l'entendement bumain de Huet et l'effet produit dans la République des Lettres en 1718 par l'édition de Fabricius des Hypotyposes de Sextus Empiricus, traduite en français par Claude Huart sept ans plus tard. Mais Popkin se refuse à penser que le scepticisme ait pu être un des traits dominants d'un siècle placé sous le signe du progrès des connaissances et des réalisations techniques, évolution exprimée dans toute sa quintessence par le projet encyclopédique de Diderot et d'Alembert. Et Popkin de conclure: "cette enquête sur le scepticisme des Lumières suggère, du moins en ce qui me concerne, que le XVIII ${ }^{\mathrm{e}}$ siècle se présente d'abord comme un hiatus dans le développement continuel du scepticisme ${ }^{3}$ ". Treize ans plus tard, dans un article intitulé "Scepticism and Anti-scepticism in the Latter Part of the Eighteenth Century ", la conclusion est identique, quoique plus lacunaire: "Au milieu du XVIII siècle, Hume était le seul sceptique vivant ${ }^{4} »$.

Dès lors, quel intérêt à évoquer la place du scepticisme dans la pensée de Rousseau si, d'une part, le scepticisme représente un courant de pensée anecdotique dans la philosophie des Lumières, et si, d'autre part, Rousseau, qui n'est jamais cité par Popkin, semble n'y jouer aucun rôle ? Pour y répondre, il faut d'abord opposer Popkin à lui-même en tenant compte des travaux qu'il a effectués par la suite et dans lesquels il avoue, avec l'honnêteté intellectuelle qui fut toujours la sienne, s'être trompé. Dans ce sens, je chercherai d'abord à montrer en quoi Popkin s'est trompé, et ce à partir de sa propre lecture de ses premiers errements, puis je poursuivrai l'analyse du scepticisme des

2. Ibid., p. 8 (traduction nôtre).

3. Ibid., p. 13 (traduction nôtre).

4. Popkin (1997), p. 19 (traduction nôtre). 
Lumières en évoquant quelques points que Popkin n'a pas soulevés et qui contribuent à modifier l'image que l'on s'en fait avant d'en venir plus précisément à la pensée de Rousseau et à la relation très particulière que le citoyen de Genève a entretenue avec le pyrrhonisme.

\section{Popkin contre Popkin}

C'est en 1992 que Popkin fait paraître un article intitulé " News Views on the Role of Scepticism in the Enlightenment ${ }^{5} »$ dans lequel il reconnait avec impartialité que le scepticisme des Lumières «était plus fondamental et plus subtil qu'[il] ne l'avait imaginé, en dépit de l'optimisme scientifique et de la croyance dans un possible progrès sans fin de la connaissance humaine partagés par les penseurs les plus notables de cette époque ${ }^{6}{ }$. Et de citer à ce propos les travaux de Giorgio Tonelli, Keith Baker et Ezequiel de Olaso allant dans le sens d'une réhabilitation de la dimension sceptique au sein de la pensée des Lumières ${ }^{7}$. Avant d'évoquer la relecture du scepticisme des Lumières proposée par Ezequiel de Olaso, sur laquelle je reviendrai par la suite quand j'évoquerai Rousseau, un mot rapide concernant les contributions de Tonelli et de Baker sur cette question afin d'expliciter en quoi ces travaux ont contribué à réhabiliter le scepticisme des Lumières.

Giorgio Tonelli a le double mérite d'avoir insisté sur l'importance du scepticisme d'un point de vue global ${ }^{8}$ tout en faisant sortir de l'ombre la figure de Pierre-Jacques Changeux ${ }^{9}$, auteur d'un scepticisme particulier développé dans son Traité des Extrémes ${ }^{10}$, qui, par son existence même, ruine la première thèse de Popkin voulant que Hume ait été le seul sceptique des Lumières. Pour Tonelli, la thèse défendue par Ernst Cassirer ${ }^{11}$ ou Paul Hazard ${ }^{12}$, qui fait des Lumières l'époque dans laquelle la raison humaine, en révolte contre les chaînes imposées par la tradition et l'autorité, impose ses propres règles dans l'ordre du savoir, ouvrant par là l'ère du progrès scientifique et de l'arraisonnement du monde, est totalement à reconsidérer. Le XVIII ${ }^{\mathrm{e}}$ siècle ne saurait en effet se réduire à ce schéma simplificateur qui, sous prétexte d'unifier les courants de pensée divers qui le parcourent, aplatit les idées et les concepts pour mieux en rendre compte. Ce qui ne veut bien évidemment pas signifier que cette thèse est fausse, mais simplement qu'elle ne saurait suffire à expliquer

5. Cf. Popkin (1997).

6. Ibid., p. 157 (traduction nôtre).

7. De nos jours, cette importance du scepticisme des Lumières est devenue un lieu commun. Voir à ce propos la bibliographie très complète sur le sujet que proposent Bernier et Charles (2005), pp. 248-254.

8. Voir Popkin (1997), pp. 35-50.

9. Cf. Popkin (1997), pp. 51-68.

10. Changeux (1767). Un résumé du livre de Changeux par Vallet a été publié en 1772 dans le dix-huitième volume de l'Encyclopédie d'Yverdon et a été reproduit dans le tome II du Supplément à l'Encyclopédie de Diderot en 1776 sous l'article « Extrême » (pp. 928-932).

11. Cassirer (1966).

12. Hazard (1963). 
seule toute la complexité d'une atmosphère intellectuelle spécifique, celle d'un siècle où l'on a refusé dogmatisme et esprit de système, qui ne doit pas être appréhendée à partir d'un seul angle interprétatif.

Tonelli a bien mis en avant le revers d'une telle médaille : si les Lumières représentent bien l'Âge de la Raison, il faut néanmoins préciser qu'il s'agit là d'une raison élaborant ses propres limites et établissant avec prudence le domaine du savoir humain en assignant des bornes strictes à la connaissance. Bien sûr, en magnifiant les trois héros des siècles passés (Bacon, Locke et Newton), en les présentant comme les représentants des droits de la raison, mais aussi de ses devoirs à l'égard de ses propres limites en général et de l'expérience en particulier, les philosophes des Lumières pensaient sincèrement suivre les lois de la raison et non les préjugés ou la tradition, mais ils ne faisaient en fait à leur tour que remplacer une forme d'autorité par une autre ${ }^{13}$. Il est pourtant tout à leur honneur d'avoir tenté de délimiter le champ de la rationalité humaine, limitation dont Tonelli donne sept exemples qui sont autant de thèses sceptiques à l'égard des potentialités de la raison humaine: (1) il est impossible de connaître les objets transcendant toute expérience humaine; (2) il est impossible de connaître les choses qui nous entourent telles qu'elles sont en elles-mêmes; (3) la notion de substance est métaphysiquement inconcevable; (4) la texture interne des corps et leurs propriétés les plus propres sont inconnaissables; (5) la connaissance des causes nous est refusée (ce qui n'est pas sans importance pour le traitement de l'idée de finalité); (6) le recours aux hypothèses est à privilégier et l'utilisation du système à bannir ; (7) la notion d'infini nous est inaccessible. De là, Tonelli conclut à la nécessaire réévaluation du scepticisme au XVIII ${ }^{\mathrm{e}}$ siècle, à entreprendre en conjonction avec la redéfinition par les philosophes des Lumières eux-mêmes de la nature de la métaphysique $^{14}$.

La figure de Pierre-Jacques Changeux permet une telle réévaluation puisqu'elle montre toute la prégnance du scepticisme au sein des Lumières. « Pour ma part, écrit Tonelli, l'œuvre principale de Changeux mérite quelque attention [...] parce que ce dernier est un représentant typique (et relativement tardif) du courant sceptique majoritaire propre à la philosophie française du $\mathrm{XVIII}^{\mathrm{e}}$ siècle qui a été jusqu'à présent presque totalement ignoré ${ }^{15}{ }^{\prime}$. Au-delà du coup de patte implicite à Popkin, le jugement de Tonelli est exact quant à Changeux, dont le Traité des Extrémes s'inscrit effectivement dans un courant sceptique fort, et l'article de Tonelli en rappelle les grandes figures, démarche qui lui permet de conclure en montrant que le scepticisme «était beaucoup plus largement diffusé en France à cette époque que cela a été dit jusqu'à présent, et tellement d'ailleurs qu'il est probablement justifié de le considérer comme le courrant méthodologique le plus dominant de cette époque ${ }^{16}{ }$.

\footnotetext{
13. Voir notamment à ce propos Mayer (1956).

14. Cf. Charles (2000).

15. Tonelli, dans Popkin (1997), p. 52 (traduction nôtre).

16. Ibid., p. 54 (traduction nôtre).
} 
Keith Baker, de son côté, a montré que celui qui semblait incarner la figure exemplaire de l'optimisme des Lumières, à savoir Condorcet, n'était peut-être pas aussi confiant dans les potentialités humaines qu'on aurait pu le croire au premier abord ${ }^{17}$, ce qui a conduit par la suite Popkin lui-même à voir en lui un sceptique " radical $^{18} »$ - ce qui ne contredit pas pour autant, à ses yeux, son optimisme. S'il fut un des plus grands mathématiciens de son temps et notamment l'instigateur de l'application des mathématiques aux problèmes humains par le biais des calculs de probabilité, son approche de la science mathématique est indissociable de son scepticisme méthodologique. Peut-on y lire, comme le propose Popkin, « l'épistémologie sceptique la plus avancée de tous les philosophes des Lumières ${ }^{19}$ » ? C'est passer d'un excès, l'inexistence du scepticisme des Lumières, à un autre, l'influence exceptionnelle du scepticisme sur la philosophie du XVIII ${ }^{\mathrm{e}}$ siècle. Concernant Condorcet, les choses sont plus complexes et son scepticisme est avant tout méthodologique, comme ce dernier le rappelle dans ses Notes sur Voltaire où il le présente comme un juste milieu acceptable entre dogmatisme et pyrrhonisme: " les déclamations contre le scepticisme sont l'ouvrage de la sottise ou de la charlatanerie. Un sceptique qui n'admettrait pas les différents degrés de probabilité serait un fou; un sceptique qui les admet ne diffère des dogmatiques qu'en ce qu'il cherche à démêler ces différents degrés avec plus de subtilités ${ }^{20}$ ". Si Condorcet se refuse à concevoir que la nature, dans son essence, puisse nous être accessible, il pense néanmoins que l'utilisation à bon escient des données fournies par l'expérience et le recours à la théorie mathématique probabiliste permettent d'anticiper et de prévoir là où il est impossible de déterminer avec certitude le cours des événements. Le futur, s'il ne nous est pas donné puisque la nature des choses nous est définitivement refusée, reste envisageable dans ses grandes lignes. Et cela vaut autant pour les sciences naturelles que pour les sciences morales où une certitude de la même espèce peut être envisagée (car, pour Condorcet, les mathématiques, tout comme la physique ou les sciences morales, ne sont que probables). Dès lors, les sciences peuvent construire leur objet sur un fond de scepticisme ${ }^{21}$.

Si les travaux de Tonelli et Baker ont contribué à faire rejaillir l'importance du scepticisme au XVIII ${ }^{\mathrm{e}}$ siècle, c'est avant tout un scepticisme de fin de siècle qui utilise les arguments sceptiques principalement dans une visée épistémologique. Il me semble que cette influence sceptique, à laquelle Popkin a accordé beaucoup d'importance dans ses derniers travaux, notamment en s'intéressant au Projet de scepticisme universel de Brissot de Warville écrit dans

17. Baker (1975).

18. Popkin (1998), pp. 31-39.

19. Ibid., p. 36 (traduction nôtre).

20. Condorcet, Notes sur Voltaire, dans Concorcet (1847), t. IV, p. 559.

21. Cette utilisation du calcul des probabilités à titre de science prédictive se retrouve à la même époque chez Jean-Claude de La Métherie (1787), qui propose un tableau des certitudes (t. I, pp. 466-478). 
les mêmes années et dans une perspective assez identique, ne rend compte que d'un aspect des choses et que la prégnance sceptique au siècle des Lumières, d'une part, ne se réduit pas à des penseurs comme Hume, Changeux, Condorcet ou Brissot de Warville, et que, d'autre part, elle se manifeste bien plus tôt dans le siècle.

\section{Scepticisme clandestin et égoïsme métaphysique}

Afin de valider cette interprétation, je prendrai deux exemples, ceux du scepticisme clandestin et de l'égoïsme philosophique. Si les recherches sur le scepticisme clandestin de l'âge classique en sont encore à leurs balbutiements et ne proposent pas de vision univoque du phénomène, elles s'entendent néanmoins pour insister sur l'importance du mouvement sceptique à l'intérieur de la clandestinité philosophique ${ }^{22}$. C'est le cas notamment des recherches menées par Miguel Benítez ${ }^{23}$ (qui rejoignent d'ailleurs sur certains points notre critique de la position de Popkin) dont je n'en dégagerai que les conclusions qui me paraissent les plus intéressantes.

Si l'on fait exception des Arguments $d u$ pyrrhonisme, manuscrit qui expose en détails certaines argumentations sceptiques à une demoiselle avide d'en savoir plus sur le pyrrhonisme, aucun manuscrit clandestin ne s'est donné pour ambition de fournir une vision impartiale de ce que fut le scepticisme ou de rappeler l'histoire de la secte et les enjeux propres à ce courant de pensée. Dans de nombreux manuscrits, le scepticisme apparaît comme une méthode que l'on utilise, non comme un résultat que l'on prône. Il ne s'agit pas de parvenir à un ensemble cohérent de propositions sceptiques qui fasse système, à une métaphysique, mais de se servir du scepticisme pour remettre en question les préjugés et indiquer les limites de la connaissance humaine. On comprend alors que l'épistémologie générale des manuscrits clandestins soit inspirée par l'empirisme et le nominalisme où l'on s'en tient au singulier en refusant de se prononcer sur ce qui n'est pas par nature perceptible. Ce scepticisme modéré «ne veut point s'interroger, en principe, sur les causes dernières et les essences et voit dans la raison qui s'appuie sur les données immédiates de l'expérience le moyen le plus adéquat pour obtenir les connaissances indispensables en vue d'assurer la préservation et le bonheur des hommes, d'un scepticisme enfin conçu comme méthode de recherche de la vérité et non comme système ${ }^{24}$ ».

Ce scepticisme est modéré parce qu'il accorde à titre d'évidences un certain nombre de pré-requis comme l'existence du monde, d'autrui et de soi (refus du solipsisme); la confiance dans les potentialités propres de l'expérience et de la raison; l'idée que la connaissance est bornée, mais qu'à l'intérieur de ces bornes la certitude est possible. Cette modération ne l'empêche pas de prendre parti ou bien pour l'idéalisme de type malebranchiste (comme dans

22. Cf. le chapitre consacré au scepticisme clandestin dans Paganini (2005), pp. 79-108. Voir également: Alan C. Kors (1993 et 2002); Canziani (1997); McKenna (2002).

23. Benítez (1996), notamment le chapitre 12, pp. 307-342.

24. Ibid., p. 308. 
les Réflexions morales et métaphysiques), ou bien, plus généralement, pour le matérialisme, mais un matérialisme qui n'a pas la cohérence et la consistance de celui développé par la suite par les philosophes matérialistes des Lumières. "Ce scepticisme se veut, en principe, un instrument critique dans la révision d'un savoir qui se prétend en possession d'une réponse définitive pour toutes les questions que la curiosité a enfantées dans le cœur des hommes. L'examen conduira à l'impossibilité de connaître la vraie nature des choses: si l'on ne peut pas douter de l'existence de la matière, il faut néanmoins avouer que nous ignorons et ses propriétés les plus intimes et la manière dont elle agit dans ses productions ${ }^{25} »$. On trouve une telle utilisation du scepticisme dans un certain nombre de manuscrits clandestins tels que Parité de la vie et de la mort; Dissertation sur la formation du monde; Essais sur la recherche de la vérité; Essais sur les facultés de l'âme; Dialogues sur l'âme; Lettre sur M. Locke. Dans ces différents manuscrits, on pose la matière comme substance sans pouvoir en donner l'essence et on bute sur des questions métaphysiques insolubles (éternité et infinité de la matière, origine et nature du mouvement, essence de la pensée) qui conduisent à reconnaître des bornes à la connaissance humaine. Au-delà de la certitude possible à l'intérieur des limites propres à l'entendement humain, il nous est loisible d'émettre un certain nombre d'hypothèses, de faire part de probabilités et de vraisemblances, un peu à la manière des académiciens (comme c'est le cas dans Parité de la vie et de la mort ou dans les Essais sur la recherche de la vérité), ou de glisser plus radicalement vers une position plus dogmatique, comme par exemple dans l'Essai de quelques idées de Dieu.

Ce jugement porté à l'encontre de la littérature clandestine, parce qu'il se veut général, ne tient pas compte de certains manuscrits qui ont donné au scepticisme une place bien plus centrale, tels que les Doutes des pyrrhoniens ou L'Art de ne rien croire. Difficile, face à ces deux textes, de déterminer ce qui est de l'ordre de l'artifice rhétorique, de la sincérité philosophique ou de la simple prudence méthodologique. Gianni Paganini a présenté une interprétation ontologique de même facture pour ces deux manuscrits : ils défendraient au fond une conception naturaliste ou spinoziste du divin ${ }^{26}$. Les deux manuscrits utiliseraient donc le pyrrhonisme de manière similaire et exprimeraient l'essence du scepticisme du début du XVIII ${ }^{e}$ siècle, moins soucieux d'agnosticisme et de suspension du jugement que de spinozisme et de naturalisme.

On pourrait dire que pour ces auteurs le scepticisme serait un effet de surface, mais commandé par un dogmatisme de fond où se cache une interprétation à la fois panthéiste et matérialiste du Deus sive natura [...]. Le dogmatisme qui resurgit après le traitement sceptique des croyances traditionnelles a le visage agressif et audacieux d'un déisme radical, poussé jusqu'à la limite du naturalisme

25. Ibid., p. 318.

26. Concernant les Doutes des pyrrhoniens, voir Paganini (1994 et 1997); en ce qui a trait à L'Art de ne rien croire, se référer à Paganini (2007). 
par son idée d'une divinité tout à fait impersonnelle, simple cause nécessaire qui agit par nécessité, se confondant avec l'univers qu'elle met en mouvement ${ }^{27}$.

En ce qui concerne les Doutes des pyrrhoniens, de nombreux indices textuels vont dans le sens de la thèse de Gianni Paganini et le manuscrit ne correspond pas à un traité sceptique préoccupé de suspension du jugement et d'agnosticisme. Le scepticisme y joue avant tout un rôle méthodologique afin de répondre à la question centrale du manuscrit qui est de savoir si la religion est ou non un artifice de certains hommes utilisé pour dominer les autres, et ce afin de déterminer sa fonction et son utilité précises et de voir s'il est ou non possible de s'en passer pour proposer à la place une éthique coupée de tout référentiel théologique. On comprend alors que la question d'un Dieu rémunérateur soit l'un des enjeux centraux du texte et que la mise en doute de tout ce qui pourrait en permettre l'existence (immortalité de l'âme, méchanceté absolue de l'homme qui supposerait l'existence de châtiments éternels) dessine en contrepartie la figure d'une divinité bien différente de celle tracée par les religions positives. Cette nouvelle figure du divin tend vers une sorte de spinozisme alliant panthéisme et matérialiste dans lequel Dieu se présente comme un être nécessaire et omniprésent puisque se confondant avec l'univers éternel où il s'exprime, indifférent à ce qui est et en aucune manière rémunérateur.

Le cas de L'Art de ne rien croire ou Pseudo-Vallée me semble plus difficile à interpréter et je ferai ici l'hypothèse qu'il ne rentre pas dans la catégorisation proposée par Paganini, et ce malgré les passages du manuscrit qui vont dans le sens de sa thèse. En effet, je concède sans difficulté que l'on peut y découvrir une sorte de déisme radical où Dieu produit tout, où le déterminisme universel est total et le libre arbitre une illusion, mais il me semble qu'on ne peut pas pour autant en conclure que ce déisme n'est rien d'autre qu'un naturalisme ou un matérialisme de type spinoziste où Dieu et nature seraient confondus. J'y vois plutôt un texte proprement sceptique qui mêle des aspirations pyrrhoniennes à des positions néo-académiciennes ${ }^{28}$. Ainsi, dès le départ, l'auteur semble hésiter entre une attitude zététique - il évoque à de nombreuses reprises la nécessité de poursuivre la recherche - et une position académicienne selon laquelle il faut parfois se résoudre à dire que la vérité est inconnaissable. De même est-il à certains moments contraint à la suspension du jugement et à d'autres amené à privilégier le vraisemblable ou le probable. Sa dénonciation des erreurs des sens et de la faillibilité de la raison, sa distinction entre l'apparent et le caché, sont du ressort de l'épistémologie sceptique traditionnelle. Bien sûr, l'auteur du manuscrit énonce que, malgré tout, les sens nous renseignent sur la réalité de l'être et du mouvement, mais c'est pour tout de suite ajouter que leur essence est inconnaissable; s'il mentionne qu'un certain ordre paraît manifeste dans la nature, cette affirmation est nuancée par le fait même que la cause de cet ordre est déclarée inconnaissable, que Dieu

27. Paganini (2007), p. 14.

28. Ce sentiment est partagé par Marie-Hélène Cotoni (1996). 
est envisagé comme incompréhensible. Tout ce qui est au-delà du sensible ou de l'alliance entre les sens et la raison est frappé de doute et d'incertitude.

Dans ce cas, l'épochè est la meilleure des solutions car la suspension du jugement est en vérité une ouverture à une pluralité de solutions (ce que le dogmatisme ne permet pas), et le juste milieu équivaut à une attitude qui ne saurait être condamnable si Dieu existe bel et bien, ce dernier ne pouvant nous reprocher d'être restés dans les bornes du connaître qu'il a lui-même fixées. Le meilleur culte que l'on puisse rendre à Dieu, c'est donc de douter, c'est-àdire d'accepter notre condition d'être incertains et faillibles, et ce doute porte même sur l'existence de Dieu. Car le sceptique se doit de refuser tant le dogmatisme des croyants que des athées : celui des premiers parce qu'ils pensent qu'imaginer une chose revient à la poser dans l'existence, celui des seconds parce qu'ils croient que ne pas connaître une chose est nécessairement une preuve de son inexistence.

Si le scepticisme de l'auteur du Pseudo-Vallée penche malgré tout vers le déisme, c'est un déisme non dogmatique, qui ne conserve l'idée de Dieu et celle d'une vie post-mortem que pour des raisons éthiques, persuadé que, sans elles, chacun n'agirait que selon son amour-propre, sans considération d'autrui, avec pour conséquence une vie sociale impossible - ce qui remet en question le fait qu'une république d'athées vertueux soit possible et va contre le message des Doutes des pyrrhoniens ${ }^{29}$. Quant à la sagesse qui s'affirme au final du manuscrit, elle a tout de la morale pyrrhonienne et l'on y retrouve un certain nombre de thèmes proprement sceptiques comme la relativité des valeurs sociales, l'insistance sur l'indifférence à l'égard des maux qui nous frappent et que nous ne devons pas redoubler par l'imagination (l'exemple fameux du cochon de Pyrrhon est rappelé à cet égard), l'importance de ne pas craindre la mort et de l'accepter avec constance comme le font les autres animaux, le souci de vivre au présent et de viser la tranquillité de l'âme en suivant les lois et coutumes de son pays, quitte à en passer - thématique propre au libertinage moderne - par une dissociation entre ce que l'on fait en société et ce que l'on pense en privé. Le texte se conclut par un éloge sans équivoque du scepticisme qui reprend la thèse éculée des pyrrhoniens selon laquelle le scepticisme est un discours philosophique purgatif qui a pour fonction de nous débarrasser de la philosophie elle-même pour nous réconcilier avec la vie ${ }^{30}$.

Proposer une interprétation générale du scepticisme au siècle des Lumières, c'est s'interroger sur la nature et la fonction de tels textes qui montrent précisément en quoi l'atmosphère intellectuelle du XVIII siècle était imprégnée par le scepticisme, dont la place n'a donc pas été anecdotique. J'en veux pour preuve un deuxième exemple qui concerne un autre courant semi-clandestin où est privilégiée cette fois non la critique du religieux mais celle de l'épistémologie

29. L'Art de ne rien croire, p. 116 (je remercie Alain Mothu de m'avoir fait parvenir une copie de ce manuscrit).

30. Ibid., pp. 135-136. 
de l'âge classique. Il s'agit du mouvement égoïste, que Popkin n'a pas davantage pris en considération.

Créé par Addison au début du XVIII ${ }^{e}$ siècle ${ }^{31}$, le terme égoïsme a longtemps hésité entre un sens métaphysique (l'égoïste est l'individu qui se pense le seul être existant - bref, c'est un solipsiste) et un sens moral, celui que nous connaissons tous aujourd'hui. Doté du statut de secte par tous ses adversaires, affublé de fondateurs dont l'existence et la pensée sont obscurs (Jean Brunet, Gaspard Langenhert), critiqué de toutes parts, ce mouvement semble n'avoir eu de réalité que dans l'esprit de ses détracteurs et le Père Armogathe a utilisé à bon escient à son égard l'expression de "secte fantôme ${ }^{32}$ » et a eu raison de distinguer «les grands ancêtres ", ces philosophes que l'on présente non comme les chefs de la secte, mais comme des penseurs qui ont eu l'imprudence de proposer une métaphysique idéaliste dangereuse, par négligence ou dissimulation, à partir de laquelle leurs successeurs ont su tirer leur miel, des «inconnus » (Jean Brunet, Gaspard Langenhert) qui auraient fondé, organisé, dominé, utilisé la secte. Et c'est à ces disciples qu'on s'en prendra, coupables d'avoir déformé la pensée d'un imprudent ou d'un distrait.

On agitera derrière ces ombres une foule confuse de sectateurs coupables des pires excès et couverts du nom de leurs maitres. L'Égoïsme, déjà subtil, sera diffus, et c'est dans la plus grande confusion des concepts et des systèmes que son existence sera signalée, tour à tour, dans un panorama philosophique, chez Descartes, chez Spinoza, chez Malebranche, chez Leibniz, chez Berkeley et chez Hume ${ }^{33}$.

La citation du Père Armogathe est juste, mais elle ne signale pas assez vigoureusement la singularité exceptionnelle d'un de ces grands ancêtres, à savoir Berkeley. C'est ce dernier qui, pendant trente ans, entre 1745 et 1775, va être la "bête noire " (selon l'heureuse expression de Jacques Wendel) des Philosophes. Avant 1745, Berkeley est certes connu, notamment pour son Essai pour une nouvelle théorie de la vision et son Alciphron, mais il n'est pas vraiment combattu. C'est la traduction par Gua de Malves des Dialogues entre Hylas et Philonous de 1744, rééditée en $1750^{34}$, qui va confronter les penseurs des Lumières à l'égoïsme. Durant trente ans, ils seront tous unis dans un même combat, avec des motivations diverses il est vrai, mais tous également prêt à réfuter celui que d'Alembert, dans l'article «Égoïstes » de l'Encyclopédie, a considéré comme le philosophe qui, "parmi les modernes, a fait tous ses efforts pour établir ${ }^{35}$ " cette doctrine. Dans une perspective sensualiste où la civilité est une vertu, l'égoïsme est inacceptable. Pourtant, dans une même perspective où

31. Addison, The Spectator, $\mathrm{n}^{\circ}$ 562, July 2, 1714, dans Addison (1747), pp. 35-40.

32. Armogathe (1970). Voir également le travail plus récent sur la question égoïste de Sportelli (2007).

33. Ibid., pp. 118-119.

34. La première édition des Dialogues entre Hylas et Philonous date bien de 1744 et non de 1750 comme on le pense trop souvent. Cette datation est désormais sûre depuis notre découverte d'un texte de Saverien confirmant son existence: Saverien (1753), t. I, p. 225.

35. D’Alembert, article «Égoïstes », dans D’Alembert et Diderot (1751), t. V, p. 431b. 
les sens sont à l'origine du processus de connaissance, l'égoïsme est métaphysiquement irréfutable, comme l'accorderont d'Alembert et Diderot.

Les égoïstes sont en même temps les plus extravagants des philosophes, et les plus difficiles à convaincre; car comment prouver l'existence des objets, si ce n'est par nos sensations? Et comment employer cette preuve contre ceux qui croient que nos sensations ne supposent point nécessairement qu'il y ait quelque chose hors de nous? Par quel moyen les fera-t-on passer de l'existence de la sensation à celle de l'objet ${ }^{36}$ ?

Ce moyen, les philosophes des Lumières ne l'ont pas trouvé - du moins n'en ont-ils pas trouvé de concluant, même si chacun d'eux a tenté à sa manière de résoudre le problème ${ }^{37}$. La raillerie leur est alors apparue comme la meilleure arme à opposer aux égoïstes et les philosophes des Lumières ne se sont pas privés d'un tel recours. Mais l'humour n'a jamais été une preuve argumentative et le scepticisme épistémologique prétendument engendré par l'immatérialisme berkeleyen a influencé tout le XVIII siècle, avec pour conséquence un pessimisme épistémologique qui ne pouvait que revivifier le scepticisme. L'étude de la place du scepticisme et de l'égoïsme dans l'œuvre de Rousseau en servira de confirmation.

\section{Rousseau entre scepticisme et égoïsme}

Si l'on connaît l'influence de certains manuscrits clandestins sur la pensée de Rousseau ${ }^{38}$, il est difficile de dire si la conception qu'il s'est faite du scepticisme provient de sa lecture de ceux-ci ou non. Quoi qu'il en soit, au premier abord, Rousseau semble être totalement opposé au pyrrhonisme, forme de philosophie originellement introduite par un Pyrrhon narcissique qui a voulu se distinguer du commun par une doctrine aussi absurde qu'intenable ${ }^{39}$ et que les penseurs de son temps ont reprise pour remettre en question l'ordre social et politique de l'Ancien Régime. Le sceptique moderne n'est plus en quête de vérité comme le zététique de l'Antiquité, son scepticisme n'est pas un acte de doute mais de refus et, en se faisant " un pitoyable mérite de ne rien croire ${ }^{40}$ ", il n'est rien d'autre qu'un dogmatique. D'où la célèbre expression de Rousseau : " je ne connais personne de si dogmatique que les sceptiques d'aujourd'hui ${ }^{41}$ ". Un tel mot se comprend aisément pour qui connaît l'anthropologie rousseauiste. En effet, pour Rousseau, une attitude telle que celle prônée par les sceptiques, à savoir l'épochè, n'a aucun fondement naturel. L'homme est être de croyance

36. Ibid.

37. Cf. Charles (2003).

38. C'est ce que montre la thèse de Joseph Paul Free (1935).

39. Voir à ce propos le texte explicite de la préface de Narcisse, dans Rousseau (1959), t. II, p. 965. Voir également le texte de la Nouvelle Héloïse (V, 5), dans Rousseau, ibid., t. II, p. 592 et Émile (IV), dans Rouseau, ibid., t. IV, p. 568.

40. Pensées d'un cœur droit (LXV), dans Rousseau, ibid., t. II, p. 1312 (nous n'entrerons pas ici dans le débat sur l'authenticité de ce texte).

41. Émile (manuscrit Fabre), variante (b), dans Rousseau, ibid., t. IV, p. 1283. 
et non de doute: "Le doute sur les choses qu'il importe de connaitre est un état trop violent pour l'esprit humain; il n'y résiste pas longtemps, il se décide malgré lui de manière ou d'autre, et il aime mieux se tromper que ne rien croire $^{42} "$.

Dès lors, le scepticisme tout comme l'égoïsme sont des positions intenables, du moins en tant que dogmatismes. Pour ce qui concerne le scepticisme, la démarche de Rousseau est relativement complexe, comme le montre une analyse serrée de la Profession de foi du vicaire savoyard. Qu'il faille, avec Ezequiel de Olaso ${ }^{43}$, distinguer deux scepticismes, pyrrhonien et académique, ou, avec Marc-André $\mathrm{Nadeau}^{44}$, en compter trois, scepticisme apparent, pyrrhonisme et scepticisme involontaire, cela témoigne de la richesse conceptuelle de ce texte. On y retrouve d'abord la dénonciation habituelle du scepticisme comme posture intellectuelle, puis l'amorce d'une entreprise philosophique qui n'est pas sans rappeler la démarche cartésienne destinée à établir des principes sûrs afin de parer à toute forme de scepticisme, avec pour différence essentielle que le clair et le distinct ne jouent plus le rôle du critère de l'évidence, remplacés qu'ils sont par "la sincérité du cœur ${ }^{45}$ ». De certitudes en certitudes, de l'existence du moi à celle du monde puis à celle de son créateur, la progression semble continue et le scepticisme désormais réfuté. Mais quand le domaine des vérités existentielles est délaissé pour les questions de métaphysique, de morale ou de foi, les certitudes paraissent alors moins tranchées et le doute de refaire surface. Ainsi, si l'on peut à la rigueur démontrer que pour rendre compte de la cause du mouvement des corps il faut postuler l'existence d'un être spirituel, voire prétendre en faire un dogme, rien n'est aussi simple en ce qui concerne l'explication du rapport entre la volonté et l'action physique qu'elle produit, problème sur lequel on ne peut se contenter que de vraisemblance en montrant que la thèse adverse, en l'occurrence celle des matérialistes, paraît plus invraisemblable encore. Scepticisme de type académique sans doute, qui est le résultat d'un long processus où l'on distingue ce qui peut être su de ce qui ne pourra l'être, ignorance qui n'empêche ni la réflexion ni l'action et qui permet de rejeter le scepticisme pyrrhonien comme incompatible avec la nature humaine qui ne se satisfait pas de l'incertitude absolue, conclusion où l'on reconnaît des accents humiens.

En ce qui concerne l'égoïsme, la pensée de Rousseau ne connaît pas les mêmes variations et son opposition est, là, totale. Comme pour beaucoup de philosophes des Lumières, la confrontation avec le solipsisme passe par la médiation de Berkeley. Comme dans le cas du scepticisme de Pyrrhon, Rousseau reproche en premier lieu à l'évêque de Cloyne d'avoir cherché à se mettre en valeur en inventant une philosophie totalement éloignée du sens commun. Un

42. Émile (IV), dans Rousseau, ibid., t. IV, pp. 567-568. Voir également Lettre à Voltaire, dans Rouseau, ibid., t. IV, pp. 1070-1071.

43. Ezequiel de Olaso (1988).

44. Nadeau (2006).

45. Émile (IV), dans Rousseau, ibid., t. IV, p. 570. 
changement notable dans son attitude intervient en 1761 dans la Nouvelle Héloïse. Il ne s'agit plus de dénoncer l'excès des philosophes qui veulent se démarquer les uns des autres, mais d'accepter que le solipsisme puisse avoir ses raisons d'être. Seulement, la réfutation proposée par Rousseau paraît bien mince, et la simplicité tient lieu d'argument décisif: "L'évêque de Cloyne a démontré que sans rien changer aux apparences, la matière et les corps pourraient ne pas exister; est-ce assez pour affirmer qu'ils n'existent pas ? En tout ceci la seule apparence coûte plus que la réalité; je m'en tiens à ce qui est plus simple ${ }^{46}{ }$. Rappelons en passant, à titre d'anecdote, que le même recours à la simplicité avait été évoqué au siècle précédent par l'abbé de Lannion dans ses Méditations sur la métaphysique pour montrer, à l'inverse, que l'argument de la simplicité des voies divines inclinait plutôt à penser à la haute vraisemblance de l'inexistence de la matière et des corps.

Dans l'Émile, paru un an plus tard, un affinement stratégique est perceptible, ce qui laisse penser que Rousseau a pris conscience des lacunes de sa précédente tentative de réfutation. Désormais, nulle distinction entre réalité et apparence n'est requise pour rejeter l'égoïsme. Il suffit de s'interroger sur le statut perceptif des êtres humains et d'en tirer le syllogisme suivant : " Je suis affecté sans le vouloir; je ne peux ni produire ni anéantir mes sensations; donc, je n'en suis pas maître ». On en vient à penser qu'il existe quelque chose hors de nous et peu importe qu'il s'agisse d'apparences ne renvoyant à aucun substrat ou d'un substrat dont nous ne percevons que les apparences. Allons même plus loin: si ces apparences ne sont que des idées, comme le veut Berkeley, elles n'en sont pas moins différentes de moi. Et Rousseau de conclure:

Or, tout ce que je sens hors de moi et qui agit sur mes sens, je l'appelle matière, et toutes les portions de matière que je conçois réunies en êtres individuels, je les appelle des corps. Ainsi toutes les disputes des idéalistes et des matérialistes ne signifient rien pour moi. Leurs distinctions sur l'apparence et la réalité des corps sont des chimères ${ }^{47}$.

Parce que le perçu diffère du percevant - ce sur quoi Berkeley aurait été d'accord -, la présence du monde se révèle à moi dans sa plénitude, que cette dernière soit faite de phénomènes ou de substances.

Mais en quoi une telle position sensualiste suffit-elle à réfuter le solipsisme? Si le sensualisme est premier — «J'existe et j'ai des sens par lesquels je suis affecté. Voilà la première vérité qui me frappe, et à laquelle je suis forcé d'acquiescer ${ }^{48} »-$, il ne peut être le tout de l'expérience, sinon il serait impossible de transcender le phénoménal et nous en serions réduits à l'explication de la statue animée proposée par Condillac où tout découle de la perception, facultés intellectuelles comprises. Or, Rousseau refuse une telle

46. Nouvelle Héloïse (VI, 7), dans Rousseau, ibid., t. II, p. 684.

47. Émile (IV), dans Rousseau, ibid., t. IV, p. 571. Berkeley aurait pu dire au mot près la même chose, si ce n'est qu'il n'aurait pas utilisé le mot matière mais idée.

48. Émile, dans Rousseau, ibid., t. IV, p. 570. 
conséquence au nom de la conscience, et d'une conscience percevante qui diffère de ce qu'elle perçoit, ce qui, pense-t-il, lui permet d'échapper tout à la fois à l'égoïsme et à sa conséquence solipsiste, mais aussi au matérialisme, qui est une autre forme de réductionnisme. À l'inverse des philosophes matérialistes et sensualistes, qui ne distinguent pas la conscience de ses perceptions (puisqu'ils la font émerger de ces dernières et l'y réduisent), et qui sont à jamais enfermés dans le paralogisme berkeleyen, Rousseau postule l'existence d'un sentiment interne garant à la fois de notre liberté et de notre indépendance, comme il l'explique à M. de Franquières en 1769: «Ôtez le sentiment intérieur, et je défie tous les philosophes modernes de prouver à Berkeley qu'il y a des $\operatorname{corps}^{49}$ ".

Rousseau a bien perçu le danger d'un certain cartésianisme qui, en séparant ontologiquement res extensa et res cogitans, oblige à choisir entre deux formes de monisme: un spiritualisme où la seule réalité est d'ordre intellectuel et un matérialisme où l'esprit se réduit au cerveau. Pour éviter ces deux écueils, il choisit d'en revenir au dualisme et donc à un autre cartésianisme, moins radical cette fois, sans pour autant s'interroger comme Descartes sur la possibilité d'un Malin Génie cherchant à nous tromper, Malin Génie qu'il ne peut penser puisqu'il s'agit pour lui, comme pour les hommes des Lumières, de refuser les hypothèses métaphysiques absurdes du siècle précédent. S'il ne cherche pas à prouver la réalité du dualisme par la raison, c'est qu'il la fonde sur le sentiment intérieur, sorte de profession de foi que Kant retrouvera, qui postule que les êtres humains sont des êtres moraux libres dans un monde créé par un Dieu transcendant, profession qui n'a de valeur que pour ceux qui l'éprouvent également, c'est-à-dire, au plan épistémologique, aucune... Rousseau est bien, comme le dit justement Ezequiel de Olaso, «le premier métaphysicien qui prend conscience du fait que ses thèses intègrent des antinomies insolubles pour la raison théorique ${ }^{50} »$.

Ainsi donc, Rousseau n'échappe pas au scepticisme: il compose avec et n'hésite pas à s'en servir, même s'il en refuse les conclusions les plus extrêmes et le dogmatisme possible au nom de l'existence de ce sentiment intérieur qu'une grande partie des philosophes ne lui concède pas. Il n'échappe pas plus à l'égoïsme qui force les penseurs des Lumières à se démarquer sur le terrain ontologique et à accepter telle ou telle forme de monisme ou de dualisme. De cette confrontation avec le scepticisme et l'égoïsme, Rousseau retient la nécessaire limitation du savoir humain et la nécessité de se positionner malgré tout dans un monde incertain à partir de principes auxquels on ne peut accorder que vraisemblance et non certitude. Scepticisme mitigé sans doute, mais scepticisme tout de même, que l'on peut retrouver à l'identique ou avec des variantes plus ou moins prononcées chez de nombreux autres penseurs des Lumières et qui rend irréaliste la vision d'un XVIII ${ }^{\mathrm{e}}$ siècle optimiste et serein, au sein

49. Lettre à M. de Franquières, dans Rousseau, ibid., t. IV, p. 1139.

50. Olaso (1988), p. 22 (traduction nôtre). 
duquel une rationalité trop sûre d'elle-même est en marche vers un progrès indéfini et l'homme vers une perfectibilité tant physique que morale. Au milieu du XVIII ${ }^{e}$ siècle, Hume était donc loin d'être le seul sceptique vivant ...

\section{Bibliographie:}

L'Art de ne rien croire (édition d'Alain Mothu d'après le manuscrit de Hambourg à paraître chez Honoré Champion).

Addison, J. The Spectator, Londres, J. et R. Tonson et S. Draper, 1747, 8 t.

Alembert, J. et D. Diderot, dir., Encyclopédie ou Dictionnaire raisonné des sciences, des arts et des métiers, Paris, 1751-1765, $17 \mathrm{t}$.

—. dir., Supplément à l'Encyclopédie, Amsterdam, M.M. Rey, 1776-1777, 3 t.

Armogathe, J.-R. Une secte fantôme au dix-huitième siècle: les Égoïstes, Paris, 1970 (exemplaire disponible à la bibliothèque de l'École Normale Supérieure, côte Mémoires 1970_15).

Baker, K. Condorcet: From Natural Philosophy to Social Mathematics, Chicago, University of Chicago Press, 1975.

Benítez, M. La face cachée des Lumières, Paris/Oxford, Universitas/Voltaire Foundation, 1996.

Bernier, M. A. et S. Charles, dir., Scepticisme et Modernité, Saint-Étienne, Publications de l'Université de Saint-Étienne, 2005.

Canziani, G. «Scepticisme et religion dans le Symbolum sapientiae ", La Lettre clandestine, 6, 1997, pp. 173-187.

Cassirer, E. La philosophie des Lumières (traduction de Pierre Quillet), Paris, Fayard, 1966.

Changeux, P.-J. Traité des Extrémes, ou des élémens de la science de la réalité, Amsterdam, 1767, 2 t.

Charles, S. Berkeley au siècle des Lumières. Immatérialisme et scepticisme an XVIII siècle, Paris, Vrin, 2003.

—_. "Entre roman et histoire: la métaphysique au siècle des Lumières ", dans L. Langlois et J.-M. Narbonne, dir., Actes du XXVII Congrès de l'Association des Sociétés de Philosophie de Langue Française, Paris/Sainte-Foy, Vrin/Presses de l'Université Laval, 2000, pp. 337-345.

Condorcet, J. A. N. Euvres de Condorcet, Paris, Firmin Didot frères, 1847-1849, 12 t.

Cotoni, M.-H. « La notion d'incertitude dans l'examen des religions, du Pseudo-Vallée à Voltaire », La Lettre clandestine, 5, 1996, pp. 333-352.

Free, J. P. Rousseau's Use of the Examen de la Religion and of the Lettre de Thrasybule à Leucippe, Princeton, 1935 (thèse de doctorat).

Hazard, P. La pensée européenne au XVIII siècle de Montesquieu à Lessing, Paris, Fayard, 1963.

Kors, A. C. "Skepticism and the Problem of Atheism in Early-Modern France ", dans R. H. Popkin et A. Vanderjagt, dir., Scepticism and Irreligion in the Seventeenth and Eighteenth Centuries, Leiden, Brill, 1993, pp. 185-215.

—. "Skepticism and Clandestinity », dans G. Paganini, M. Benítez et J. Dybikowski, dir., Scepticisme, clandestinité et libre pensée, Paris, Champion, 2002, pp. 4765 . 
La Métherie, J.-C. Principes de la philosophie naturelle, dans lesquels on cherche à déterminer les degrés de certitude ou de probabilité des connoissances humaines, Genève, 1787, 2 t.

Mayer, J. "Illusions de la philosophie expérimentale au XVIII ${ }^{\mathrm{e}}$ siècle », Revue générale des sciences pures et appliquées, 63, 1956, pp. 353-363.

McKenna, A. "Le ver est dans le fruit: le scepticisme au XVIII siècle : l'exemple de Delaube », dans G. Paganini, M. Benítez et J. Dybikowski, dir., Scepticisme, clandestinité et libre pensée, Paris, Champion, 2002, pp. 165-177.

Nadeau, M.-A. "Le scepticisme de Rousseau dans La profession de foi du vicaire savoyard», Lumen, 25, 2006, pp. 29-40.

Olaso, E. "Los dos escepticismos del vicario saboyano ", (1980), réédité dans R. A. Watson et J. E. Force, dir., The Sceptical Mode in Modern Philosophy. Essays in Honor of Richard H. Popkin, Dordrecht, Kluwer, 1988, pp. 43-57.

Paganini, G. Les philosophies clandestines, Paris, Presses Universitaires de France, 2005.

_.. "Scepsi clandestina : i Doutes des pyrrhoniens », dans G. Canziani, dir., Filosofia e religione nella letteratura clandestina, Milan, Franco Angeli, 1994, pp. 83-122.

- «Du bon usage du scepticisme : les Doutes des pyrrhoniens », dans A. McKenna et A. Mothu, dir., La philosophie clandestine à l'âge classique, Paris/Oxford, Universitas/Voltaire Foundation, 1997, pp. 291-306.

- - "Avant la Promenade du sceptique: pyrrhonisme et clandestinité de Bayle à Diderot », dans G. Paganini, M. Benítez et J. Dybikowski, dir., Scepticisme, clandestinité et libre pensée, Paris, Champion, 2002, pp. 17-46.

- _L'apport des courants sceptiques à la naissance des Lumières radicales ", in C. Secrétan, T. Dagron et L. Boye (dirs.), Qu'est-ce que les Lumières « radicales»?, Paris, Éditions Amsterdam, 2007, p. 87-103.

Popkin, R. H. "Scepticism in the Enlightenment », dans R. H. Popkin et al, dir., Scepticism in the Enlightenment, Dordrecht, Kluwer, 1997, pp. 1-16.

_. "News Views on the Role of Scepticism in the Enlightenment ", dans R. H. Popkin et al, dir., Scepticism in the Enlightenment, Dordrecht, Kluwer, 1997, pp. 157172.

- "Scepticism and Anti-Scepticism in the Latter Part of the 18th Century ", dans R. H. Popkin et al, dir., Scepticism in the Enlightenment, Dordrecht, Kluwer, 1997, pp. 17-34.

—. «Brissot and Condorcet : Skeptical Philosophers ", dans R. H. Popkin et J. Van der Zande, dir., The Skeptical Tradition around 1800. Skepticism in Philosophy, Science, and Society, Dordrecht, Kluwer, 1998, pp. 31-39.

Rousseau, J.-J. CEuvres complètes (édition de Bernard Gagnebin et Marcel Raymond), Paris, Gallimard, 1959-1995, 5 t.

Saverien, A. Dictionnaire universel de Mathématique et de Physique, Paris, Rollin et Jombert, 1753, 2 t.

Tonelli, G. "The 'Weakness' of Reason in the Age of Enlightenment » (1971), dans R. H. Popkin et al, dir., Scepticism in the Enlightenment, Dordrecht, Kluwer, 1997, pp. 35-50.

_- «Pierre-Jacques Changeux and Scepticism in the French Enlightenment » (1974), dans R. H. Popkin et al, dir., Scepticism in the Enlightenment, Dordrecht, Kluwer, 1997, pp. 51-68. 